\title{
Analytical study of the dynamic behavior of geometrically nonlinear shaft-disk rotor systems
}

\author{
Muhammad Rizwan Shad ${ }^{1}$, Guilhem Michon ${ }^{2, a}$ and Alain Berlioz ${ }^{3}$ \\ 1 Université de Toulouse, ICA, INSA, 135 Av. de Rangueil, 31077 Toulouse, France \\ 2 Université de Toulouse, ICA, ISAE, 10 Av. Édouard Belin, 31055 Toulouse, France \\ 3 Université de Toulouse, ICA, UPS, 118 route de Narbonne, 31062 Toulouse, France
}

Received 6 December 2010, Accepted 21 August 2011

\begin{abstract}
This paper explores analytically the nonlinear dynamic behavior of rotors. Coupled nonlinear equations of motion are formulated using Hamilton's principle. The rotor model is composed of a rigid disk and a flexible shaft which is characterized as a beam of circular cross section. Various influences are taken into account like the effect of higher order large deformations, rotary inertia, gyroscopic effect, rotor unbalance and the effect of a dynamic axial force. Forced response due to a mass unbalance is presented first for the linear analysis and then perturbation techniques are used to solve the complete equations of motion including nonlinear terms. Method of multiple scales is applied to examine the nonlinear behaviour of the rotor system. Resonant curves are plotted for different possible resonance conditions. It is concluded that the higher order large deformations and axial force acting dynamically on the rotor have a significant effect on its nonlinear response. This response varies for different parameters of the rotor like an unbalance mass and diameter of the shaft.
\end{abstract}

Key words: Rotordynamics / nonlinear / large deformations in bending / method of multiple scales / resonant curves

Résumé - Étude analytique du comportement dynamique de systèmes tournants à nonlinéarités géométriques. Cet article est une contribution à l'étude analytique du comportement dynamique non-linéaire d'un rotor. Le principe de Hamilton est utilisé pour développer un modèle mathématique composé d'équations différentielles non-linéaires couplées du second ordre. La géométrie du rotor comprend un disque rigide et un arbre flexible modélisé par une poutre de section circulaire. Plusieurs effets sont pris en compte : les grandes déformations, l'inertie de rotation, l'effet gyroscopique, le balourd et l'effort axial dynamique. La réponse forcée provoquée par le balourd est décrite pour les analyses linéaires et non-linéaires. Une technique de perturbation, la méthode des échelles multiples, est appliquée pour étudier le comportement non-linéaire du système. L'influence de différents paramètres sur le comportement non-linéaire du rotor est présentée et discutée. Il est montré que les grandes déformations et l'effort axial dynamique appliqué au rotor ont un impact significatif sur sa réponse non-linéaire. Cette réponse est étudiée pour plusieurs paramètres du rotor tels que le balourd et le diamètre de l'arbre.

Mots clés : Dynamique des rotors / non-linéaire / grandes déformations en flexion / méthode des echelles multiples / courbes de résonance

\section{Introduction}

Investigations of the dynamic behavior of the rotor systems have been of great interest for many years. For example, numerous books have been dedicated to this topic; see for example [1-7], where all the authors have principally dealt with linear systems except

${ }^{a}$ Corresponding author: guilhem.michon@isae.fr
Yamamoto and Ishida [4] who have also treated nonlinear rotating shaft and Jeffcott rotor. Batko et al. [8] consider the effect of an axial force. During recent years, the increasing need of optimized performance of rotating machinery such as jet engines, helicopter rotors, gas turbines, steam turbines, reciprocating and centrifugal compressors, the spindle of machine tools, has added to the importance of considering nonlinear effects on their dynamics. With the ongoing research, this field has become more 


\section{Nomenclature}

\begin{tabular}{|lll|}
\hline$A$ & Cross sectional area of the shaft & $\mathrm{m}^{2}$ \\
$c$ & Coefficient of damping & $\mathrm{N} \cdot \mathrm{s}^{-1}$ \\
$d_{1}$ & Position of mass unbalance from geometric center of the shaft & $\mathrm{m}$ \\
$i$ & $\sqrt{-1}$ & \\
$I$ & Area moment of inertia of the shaft & $\mathrm{m}^{4}$ \\
$I_{\mathrm{d} x}$ & Area moment of inertia of the shaft in the $X$ direction & $\mathrm{m}^{4}$ \\
$I_{\mathrm{d} x}$ & Area moment of inertia of the shaft in the $Y$ direction & $\mathrm{m}^{4}$ \\
$L$ & Length of the shaft & $\mathrm{m}$ \\
$l_{1}$ & Position of the disk on the shaft & $\mathrm{m}$ \\
$M_{\mathrm{d}}$ & Mass of the disk & $\mathrm{kg}$ \\
$M_{\mathrm{u}}$ & Mass unbalance & $\mathrm{kg}$ \\
$R_{1}$ & Radius of the shaft/internal radius of the disk & $\mathrm{m}$ \\
$R_{2}$ & External radius of the disk & $\mathrm{m}$ \\
$T_{\mathrm{s}}$ & Kinetic energy of the shaft & $\mathrm{N} \cdot \mathrm{m}$ \\
$T_{\mathrm{d}}$ & Kinetic energy of the disk & $\mathrm{N} \cdot \mathrm{m}$ \\
$T_{\mathrm{u}}$ & Kinetic energy of the mass unbalance & $\mathrm{N} \cdot \mathrm{m}$ \\
$T_{\mathrm{R}}$ & Kinetic energy of the rotor & $\mathrm{N} . \mathrm{m}$ \\
$U_{\mathrm{R}}$ & Deformation energy of the rotor & $\mathrm{N} \cdot \mathrm{m}$ \\
$U_{\mathrm{S}}$ & Deformation energy of the shaft & $\mathrm{N} . \mathrm{m}$ \\
$\rho$ & Density of the material & $\mathrm{kg} \cdot \mathrm{m}$ \\
$\Omega$ & Speed of rotation of the rotor & rad.s \\
$\omega_{1}, \omega_{2}$ & Natural frequencies of the rotor & $\mathrm{Hz}$ \\
$\sigma_{1}$ & Detuning parameter & $\mathrm{Hz}$ \\
$u(y, t)$ & Displacement along $x$ axis of the rotor & $\mathrm{m}$ \\
$w(y, t)$ & Displacement along $z$ axis of the rotor & $\mathrm{m}$ \\
$U$ & Discretized displacement along $x$ axis & $\mathrm{m}$ \\
$W$ & Discretized displacement along $z$ axis & $\mathrm{m}$ \\
$N_{\mathrm{A}}$ & Dynamic axial force & $\mathrm{N}$ \\
\hline & & \\
\hline
\end{tabular}

challenging because the analysis of nonlinear phenomena is far more difficult compared to the linear phenomenon. In a recent paper, Ehrich [9] depicted interesting phenomenon such as relaxation, sub-harmonic response and chaotic behavior that are due to nonlinearities, clearance in a ball bearing and clearance in a squeeze-film damper bearing. These phenomenon are, among other things, due to higher order large deformations and geometric nonlinearity [10], rotor-base movements $[11,12]$. Furthermore there are various secondary effects that should be considered for a complete and accurate analysis such as rotary inertia, gyroscopic effect and rotor unbalance. In addition, since a rotor executes a whirling motion due to gyroscopic moment, analytical methods used in the analysis of rectilinear systems cannot be applied directly to rotor systems.

The present work is distinguished from the previously reported works as it examines the geometrically nonlinear dynamics of shaft-disk rotor systems. Also if the supports of the rotor are such that they do not allow the shaft to move in the axial direction then there will be dynamic force acting axially on the rotor as it operates. The first part of the paper is related to the development of a mechanical model using Euler-Bernoulli beam configuration and taking into account rotary inertia and gyroscopic effects. Hamilton's principle [13] is used to derive the equations of motion. The linear part of the model developed is solved for the first mode to obtain the natural frequencies of vibrations and Campbell diagram is plotted to determine the critical speeds. Then, in a second section, in order to solve the complete model including nonlinear terms, the method of multiple scales (MMS) is applied on a discretized dynamic system. This method has been proven very effective in solving the nonlinear equations of motion particularly in the stability analysis [14-19]. The last part is devoted to the application on an academic rotor system. The responses are studied for different parameters of the rotor like an unbalance mass and diameter of the shaft.

\section{Derivation of equations of motion}

The equations of motion are derived by considering the disk of the rotor to be rigid and the shaft to be flexible. The geometry of the rotor is shown in Figure 1. Duchemin [11] have used similar rotor geometry to study the dynamics of rotors subjected to base movements. The shaft is supposed to be a flexible beam of circular cross section having length $L$ and radius $R_{1}$. It is modeled by its kinetic and strain energies denoted by $T_{\mathrm{s}}$ and $U_{\mathrm{s}}$ respectively. The disk of external radius $R_{2}$ and internal radius $R_{1}$ positioned at a distance $y=L / 3$ is considered to be rigid and hence only requires kinetic energy for its characterization denoted by $T_{\mathrm{d}}$. There is also a small unbalanced mass denoted by $m_{\mathrm{u}}$ and is located at a distance $y=L / 3$. It is described by its kinetic energy $T_{\mathrm{u}}$. 


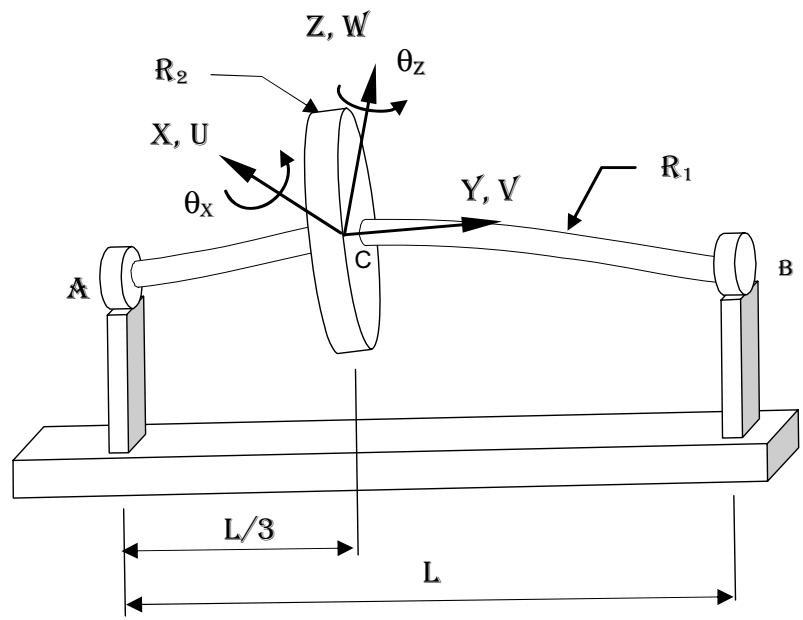

Fig. 1. Rotor with shaft and disk.

\subsection{Kinetic energy}

All the components of the rotor possess their individual kinetic energies. The expressions of these energies are given according to [2] and are reproduced as below:

$$
\begin{aligned}
T_{\mathrm{d}}= & \frac{1}{2} M_{\mathrm{d}}\left(\dot{u}^{2}+\dot{w}^{2}\right)+\frac{1}{2} I_{\mathrm{d} x}\left(\dot{\theta}_{x}^{2}+\dot{\theta}_{z}^{2}\right)+I_{\mathrm{d} y} \Omega \dot{\theta}_{z} \theta_{x} \\
T_{\mathrm{s}}= & \int_{0}^{L} \frac{\rho A}{2}\left(\dot{u}^{2}+\dot{w}^{2}\right) \mathrm{d} y+\int_{0}^{L} \frac{\rho I}{2}\left(\dot{\theta}_{x}^{2}+\dot{\theta}_{z}^{2}\right) \mathrm{d} y \\
& +\int_{0}^{L} 2 \rho I \Omega \dot{\theta}_{z} \theta_{x} \mathrm{~d} y \\
T_{\mathrm{u}}= & m_{\mathrm{u}} \Omega d_{1}(\dot{u} \cos \Omega t-\dot{w} \sin \Omega t)
\end{aligned}
$$

where, $T_{\mathrm{d}}$ is the kinetic energy of the disk and $T_{\mathrm{u}}$ represents the kinetic energy of the mass unbalance.

\subsection{Deformation energy}

Since the shaft is considered to be flexible, therefore it contains deformation energy also. This energy consists of two components:

$$
U_{\mathrm{s}}=U_{\mathrm{s} 1}+U_{\mathrm{s} 2}
$$

The first one denoted by $U_{\mathrm{s} 1}$ is due to the consideration of higher order large deformations and is given as below,

$$
\begin{aligned}
U_{\mathrm{s} 1}=\frac{E I}{2} \int_{0}^{L}\left\{\left(\frac{\partial \theta_{x}}{\partial y}\right)^{2}\right. & \left.+\left(\frac{\partial \theta_{z}}{\partial y}\right)^{2}\right\} \mathrm{d} y \\
& +\frac{E A}{2} \int_{0}^{L}\left(\frac{\theta_{x}^{4}}{4}+\frac{\theta_{x}^{2} \theta_{z}^{2}}{2}+\frac{\theta_{z}^{4}}{4}\right) \mathrm{d} y
\end{aligned}
$$

The detailed derivation of the above expression can be found in Appendix A.

The 2nd component of the deformation energy is denoted by $U_{\mathrm{s} 2}$ and is justified because of the inclusion of a dynamic axial force $N_{\mathrm{A}}$. This force will be continuously acting on the rotor if the bearings at both ends of the shaft block its axial elongation while the shaft is in bending. $U_{\mathrm{s} 2}$ is given by the following expression,

$$
U_{\mathrm{s} 2}=\frac{N_{\mathrm{A}}}{2} \int_{0}^{L}\left(\theta_{x}^{2}+\theta_{z}^{2}\right) \mathrm{d} y
$$

where $N_{\mathrm{A}}$ can be shown to be $N_{\mathrm{A}}=\int_{0}^{L} \frac{E A}{2 L}\left(\theta_{x}^{2}+\theta_{z}^{2}\right) \mathrm{d} y$.

Therefore the overall deformation energy of the shaft becomes,

$$
\begin{aligned}
U_{\mathrm{s}}= & \frac{E I}{2} \int_{0}^{L}\left\{\left(\frac{\partial \theta_{x}}{\partial y}\right)^{2}+\left(\frac{\partial \theta_{z}}{\partial y}\right)^{2}\right\} \mathrm{d} y \\
& +\frac{E A}{2} \int_{0}^{L}\left(\frac{\theta_{x}^{4}}{4}+\frac{\theta_{x}^{2} \theta_{z}^{2}}{2}+\frac{\theta_{z}^{4}}{4}\right) \mathrm{d} y \\
& +\int_{0}^{L} \int_{0}^{L} \frac{E A}{4 L}\left(\theta_{x}^{2}+\theta_{z}^{2}\right) \mathrm{d} y\left(\theta_{x}^{2}+\theta_{z}^{2}\right) \mathrm{d} y
\end{aligned}
$$

Finally, taking into account the energies of the various components of the rotor as discussed above, the total kinetic and deformations energies of the complete rotor system denoted by $T_{\mathrm{R}}$ and $U_{\mathrm{R}}$ respectively can be written as the following two equations:

$$
\begin{aligned}
T_{\mathrm{R}}=T_{\mathrm{s}}+ & T_{\mathrm{d}}+T_{\mathrm{u}} \\
= & \frac{1}{2} M_{\mathrm{d}}\left(\dot{u}^{2}+\dot{w}^{2}\right)+\int_{0}^{L} \frac{\rho A}{2}\left(\dot{u}^{2}+\dot{w}^{2}\right) \mathrm{d} y \\
& +\int_{0}^{L} \frac{\rho I}{2}\left(\dot{\theta}_{x}^{2}+\dot{\theta}_{z}^{2}\right) \mathrm{d} y+\int_{0}^{L} 2 \rho I \Omega \dot{\theta}_{z} \theta_{x} \mathrm{~d} y \\
+ & \frac{1}{2} I_{\mathrm{d} x}\left(\dot{\theta}_{x}^{2}+\dot{\theta}_{z}^{2}\right)+I_{\mathrm{d} y} \Omega \dot{\theta}_{z} \theta_{x}+m_{\mathrm{u}} \Omega d_{1} \\
U_{\mathrm{R}}= & U_{\mathrm{s}} \times(\dot{u} \cos \Omega t-\dot{w} \sin \Omega t) \\
= & \int_{0}^{L} \frac{E I}{2}\left\{\left(\frac{\partial \theta_{x}}{\partial y}\right)^{2}+\left(\frac{\partial \theta_{z}}{\partial y}\right)^{2}\right\} \mathrm{d} y \\
& +\int_{0}^{L} \frac{E A}{2}\left(\frac{1}{4} \theta_{x}^{4}+\frac{1}{2} \theta_{x}^{2} \theta_{z}^{2}+\frac{1}{4} \theta_{z}^{4}\right) \mathrm{d} y \\
& +\int_{0}^{L} \int_{0}^{L} \frac{E A}{4 L}\left(\theta_{x}^{2}+\theta_{z}^{2}\right) \mathrm{d} y\left(\theta_{x}^{2}+\theta_{z}^{2}\right) \mathrm{d} y
\end{aligned}
$$




\subsection{Discretization of equations}

Previous energy equations are composed of partial differential terms. Although it is possible to treat them in this direct form, it is preferable to use an approximation of the solutions with a variable separation technique. Therefore the equations become ordinary differential equations. Displacements $u$ and $w$, functions of $y$ and $t$, are written as the product of a function of time by a function of $y$. The expression for the displacement in $x$ and $z$ directions is that of the simply supported beam in bending:

$$
\begin{gathered}
u(y, t)=f(y) U(t)=\sin \left(\frac{\pi y}{L}\right) U \\
w(y, t)=f(y) W(t)=\sin \left(\frac{\pi y}{L}\right) W
\end{gathered}
$$

Also,

$$
\begin{gathered}
\theta_{x}=\frac{\partial w}{\partial y}=\frac{\mathrm{d} f(y)}{\mathrm{d} y} W=g(y) W \\
\theta_{z}=-\frac{\partial u}{\partial y}=-\frac{\mathrm{d} f(y)}{\mathrm{d} y} U=-g(y) U \\
\frac{\partial \theta_{x}}{\partial y}=\frac{\mathrm{d}^{2} f(y)}{\mathrm{d} y^{2}} W=h(y) W \\
\frac{\partial \theta_{z}}{\partial y}=-\frac{\mathrm{d}^{2} f(y)}{\mathrm{d} y^{2}} U=-h(y) U
\end{gathered}
$$

where, $\theta_{x}$ and $\theta_{z}$ are the angular displacements used in classical Euler-Bernoulli beam theory. By the above expressions the kinetic energy of the rotor system is given as,

$$
\begin{aligned}
T_{\mathrm{R}}=\frac{1}{2} b_{1}\left(\dot{U}^{2}+\dot{W}^{2}\right)-\Omega b_{2} \dot{U} W & +m_{\mathrm{u}} \Omega d_{1} f\left(l_{1}\right) \\
& \times(\dot{U} \cos \Omega t-\dot{W} \sin \Omega t)
\end{aligned}
$$

where,

$$
\begin{aligned}
& b_{1}=M_{\mathrm{D}} f^{2}\left(l_{1}\right)+I_{\mathrm{D} x} g^{2}\left(l_{1}\right)+\rho S \int_{0}^{L} f^{2}(y) \mathrm{d} y+\rho I \int_{0}^{L} g^{2}(y) \mathrm{d} y \\
& b_{2}=I_{\mathrm{D} y} g^{2}\left(l_{1}\right)+2 \rho I \int_{0}^{L} g^{2}(y) \mathrm{d} y
\end{aligned}
$$

Similarly, the deformation energy of the rotor is,

$$
U_{\mathrm{R}}=\frac{k_{1}}{2}\left(U^{2}+W^{2}\right)+\left(\frac{k_{2}}{8}+\frac{k_{3}}{4}\right)\left(U^{2}+W^{2}\right)^{2}
$$

where,

$$
\begin{aligned}
& k_{1}=E I \int_{0}^{L} h^{2}(y) \mathrm{d} y ; \quad k_{2}=E A \int_{0}^{L} g^{4}(y) \mathrm{d} y \\
& k_{3}=E A / L \int_{0}^{L} \int_{0}^{L} h^{4}(y) \mathrm{d} y \mathrm{~d} y
\end{aligned}
$$

\subsection{Application of Hamilton's principle}

The principle allows writing the following expression

$$
\int_{t_{1}}^{t_{2}} \delta\left(T_{\mathrm{R}}-U_{\mathrm{R}}\right) \mathrm{d} t=0
$$

Further it can be expanded as,

$$
\int_{t_{1}}^{t_{2}} \delta\left(T_{\mathrm{R}}-U_{\mathrm{R}}\right) \mathrm{d} t=\int_{t_{1}}^{t_{2}} \delta T_{\mathrm{R}} \mathrm{d} t-\int_{t_{1}}^{t_{2}} \delta U_{\mathrm{R}} \mathrm{d} t=0
$$

The first term in the above equation is sum of three terms as can be seen in the following expression,

$$
\int_{t_{1}}^{t_{2}} \delta T_{\mathrm{R}} \mathrm{d} t=\int_{t_{1}}^{t_{2}}\left[\frac{\partial T_{\mathrm{R}}}{\partial W} \delta W+\frac{\partial T_{\mathrm{R}}}{\partial \dot{U}} \delta \dot{U}+\frac{\partial T_{\mathrm{R}}}{\partial \dot{W}} \delta \dot{W}\right] \mathrm{d} t
$$

Various terms in the above equation can be calculated as follows,

$$
\begin{aligned}
& \int_{t_{1}}^{t_{2}} \frac{\partial T_{\mathrm{R}}}{\partial W} \delta W \mathrm{~d} t=-\int_{t_{1}}^{t_{2}} \Omega b_{2} \dot{U} \delta W \mathrm{~d} t \\
& \int_{t_{1}}^{t_{2}} \frac{\partial T_{\mathrm{R}}}{\partial \dot{U}} \delta \dot{U} \mathrm{~d} t=\left[\frac{\partial T_{\mathrm{R}}}{\partial \dot{U}} \delta U\right]_{t_{1}}^{t_{2}}-\int_{t_{1}}^{t_{2}} \frac{\partial}{\partial t}\left(\frac{\partial T_{\mathrm{R}}}{\partial \dot{U}}\right) \delta U \mathrm{~d} t \\
&= {\left[\frac{\partial T_{\mathrm{R}}}{\partial \dot{U}} \delta U\right]_{t_{1}}^{t_{2}}-\int_{t_{1}}^{t_{2}} \frac{\partial}{\partial t}\left(b_{1} \dot{U}-\Omega b_{2} W\right.} \\
&\left.+m_{\mathrm{u}} \Omega d_{1} f\left(l_{1}\right) \cos \Omega t\right) \delta U \mathrm{~d} t \\
& \int_{t_{1}}^{t_{2}} \frac{\partial T_{\mathrm{R}}}{\partial \dot{W}} \delta \dot{W} \mathrm{~d} t=\left[\frac{\partial T_{\mathrm{R}}}{\partial \dot{W}} \delta W\right]_{t_{1}}^{t_{2}}-\int_{t_{1}}^{t_{2}} \frac{\partial}{\partial t}\left(\frac{\partial T_{\mathrm{R}}}{\partial \dot{W}}\right) \delta W \mathrm{~d} t \\
&=\left[\frac{\partial T_{\mathrm{R}}}{\partial \dot{W}} \delta W\right]_{t_{1}}^{t_{2}}-\int_{t_{1}}^{t_{2}} \frac{\partial}{\partial t}\left(b_{1} \dot{W}-m_{\mathrm{u}} \Omega d_{1} f\left(l_{1}\right)\right. \\
&\times \sin \Omega t) \delta W \mathrm{~d} t
\end{aligned}
$$

Similarly the second term in Equation (1) gives

$$
\int_{t_{1}}^{t_{2}} \delta U_{\mathrm{R}} \mathrm{d} t=\int_{t_{1}}^{t_{2}}\left[\frac{\partial U_{\mathrm{R}}}{\partial U} \delta U+\frac{\partial U_{\mathrm{R}}}{\partial W} \delta W\right] \mathrm{d} t
$$

The two terms in the above equation can be written as below

$$
\begin{aligned}
& \int_{t_{1}}^{t_{2}} \frac{\partial U_{\mathrm{R}}}{\partial U} \delta U \mathrm{~d} t=\int_{t_{1}}^{t_{2}}\left[k_{1} U+\left(\frac{k_{2}}{2}+k_{3}\right)\left(U^{3}+U W^{2}\right)\right] \delta U \mathrm{~d} t \\
& \int_{t_{1}}^{t_{2}} \frac{\partial U_{\mathrm{R}}}{\partial W} \delta W \mathrm{~d} t=\int_{t_{1}}^{t_{2}}\left[k_{1} W+\left(\frac{k_{2}}{2}+k_{3}\right)\left(W^{3}+U^{2} W\right)\right] \delta W \mathrm{~d} t
\end{aligned}
$$


Finally, all the terms of the type $\delta U \mathrm{~d} t$ can be collected as follows

$$
\begin{aligned}
-\int_{t_{1}}^{t_{2}} \frac{\partial}{\partial t}\left(b_{1} \dot{U}-\Omega b_{2} W+m_{\mathrm{u}} \Omega d_{1} f\left(l_{1}\right) \cos \Omega t\right) \delta U \mathrm{~d} t \\
\quad-\int_{t_{1}}^{t_{2}}\left[k_{1} U+\left(\frac{k_{2}}{2}+k_{3}\right)\left(U^{3}+U W^{2}\right)\right] \delta U \mathrm{~d} t=0
\end{aligned}
$$

or

$$
\begin{array}{r}
b_{1} \ddot{U}-\Omega b_{2} \dot{W}+k_{1} U+\left(\frac{k_{2}}{2}+k_{3}\right)\left(U^{3}+U W^{2}\right) \\
=m_{u} \Omega^{2} \mathrm{~d}_{1} f\left(l_{1}\right) \sin (\Omega t)
\end{array}
$$

Similarly by collecting all the terms of the type $\delta W \mathrm{~d} t$ following equations are obtained.

$$
\begin{array}{r}
-\int_{t_{1}}^{t_{2}} \frac{\partial}{\partial t}\left(b_{1} \dot{W}-m_{\mathrm{u}} \Omega d_{1} f\left(l_{1}\right) \sin \Omega t\right) \delta W \mathrm{~d} t-\int_{t_{1}}^{t_{2}} \Omega b_{2} \dot{U} \delta W \mathrm{~d} t \\
\quad-\int_{t_{1}}^{t_{2}}\left[k_{1} W+\left(\frac{k_{2}}{2}+k_{3}\right)\left(W^{3}+U^{2} W\right)\right] \delta W \mathrm{~d} t=0
\end{array}
$$

or

$$
\begin{array}{r}
b_{1} \ddot{W}+\Omega b_{2} \dot{U}+k_{1} W+\left(\frac{k_{2}}{2}+k_{3}\right)\left(W^{3}+U^{2} W\right) \\
=m_{\mathrm{u}} \Omega^{2} d_{1} f\left(l_{1}\right) \cos \Omega t
\end{array}
$$

Equations (2) and (3) can be further written as,

$$
\begin{gathered}
\ddot{U}-\Omega \alpha_{1} \dot{W}+\alpha_{2} U+\left(\frac{\beta_{1}}{2}+\beta_{2}\right)\left(U^{3}+U W^{2}\right)+c \dot{U} \\
=m_{1} \Omega^{2} d_{1} f\left(l_{1}\right) \sin (\Omega t) \\
\ddot{W}+\Omega \alpha_{1} \dot{U}+\alpha_{2} W+\left(\frac{\beta_{1}}{2}+\beta_{2}\right)\left(W^{3}+W U^{2}\right)+c \dot{W} \\
=m_{1} \Omega^{2} d_{1} f\left(l_{1}\right) \cos (\Omega t)
\end{gathered}
$$

where,

$$
\alpha_{1}=\frac{b_{2}}{b_{1}}, \alpha_{2}=\frac{k_{1}}{b_{1}}, \beta_{1}=\frac{k_{2}}{b_{1}}, \beta_{2}=\frac{k_{3}}{b_{1}}, m_{1}=\frac{m_{u}}{b_{1}}
$$

The two nonlinear second order differential Equations (4) are the equations of motion of the rotor system studied.

The linear analysis of the free undamped system is similar to [2]. The Campbell diagram, forced response of the linear system and numerical data are presented in Appendix B.

\section{Nonlinear analysis}

As the equations of motion are written as ordinary differential equations, the method of multiple scales (MMS) is applied to treat the nonlinear system [15]. This method is a well-developed tool for the analysis of nonlinear systems. See for example [18] for the detailed procedure of the application of this method. In order to apply MMS, the displacements $U$ and $W$ are expanded in a form of first order power series of $\varepsilon$,

$$
\begin{gathered}
U\left(T_{0}, T_{1}\right)=u_{0}\left(T_{0}, T_{1}\right)+\varepsilon u_{1}\left(T_{0}, T_{1}\right)=u_{0}+\varepsilon u_{1} \\
W\left(T_{0}, T_{1}\right)=w_{0}\left(T_{0}, T_{1}\right)+\varepsilon w_{1}\left(T_{0}, T_{1}\right)=w_{0}+\varepsilon w_{1}
\end{gathered}
$$

where $T_{\mathrm{n}}=\varepsilon^{n} t$ are slow time scales, $T_{1}$ being slower than $T_{0}$ and $\varepsilon$ is a small dimensionless book-keeping parameter such that $\varepsilon \ll 1$. In order to obtain the nonlinear, damping and forcing terms in the same order of $\varepsilon$ after expansion, following scaling is used in Equations (4).

$\alpha_{1}=\alpha 1, \alpha_{2}=\alpha 2, \beta_{1}=\varepsilon \beta 1, \beta_{2}=\varepsilon \beta 2$,

$$
m_{1}=\varepsilon m 1, c=\varepsilon c 1
$$

Equations (4) and (5) can now be written as

$$
\begin{gathered}
\ddot{U}(t)-\Omega \alpha 1 \dot{W}(t)+\alpha 2 U(t)+\varepsilon \frac{\beta 1}{2}\left(U(t)^{3}+U(t) W(t)^{2}\right) \\
+\varepsilon \beta 2\left(U(t)^{3}+U(t) W(t)^{2}\right)+\varepsilon c 1 \dot{U}(t) \\
-\varepsilon m 1 \Omega^{2} d_{1} f\left(l_{1}\right) \sin \Omega t=0 \\
\ddot{W}(t)+\Omega \alpha 1 \dot{U}(t)+\alpha 2 W(t)+\frac{\varepsilon \beta 1}{2}\left(W(t)^{3}+W(t) U(t)^{2}\right) \\
+\varepsilon \beta 2\left(W(t)^{3}+W(t) U(t)^{2}\right)+\varepsilon c 1 \dot{W}(t) \\
-\varepsilon m 1 \Omega^{2} d_{1} f\left(l_{1}\right) \cos \Omega t=0 \quad(6)
\end{gathered}
$$

The various time derivatives in the above equation are now written as

$$
\begin{aligned}
\frac{\mathrm{d}}{\mathrm{d} t} & =\frac{\partial}{\partial T_{0}}+\varepsilon \frac{\partial}{\partial T_{1}} \\
\frac{\mathrm{d}^{2}}{\mathrm{~d} t^{2}} & =\frac{\partial^{2}}{\partial T_{0}^{2}}+\varepsilon \frac{\partial^{2}}{\partial T_{0} \partial T_{1}}
\end{aligned}
$$


Substituting Equations (5) and (7) in Equation (8) and equating coefficients of the like powers of $\varepsilon$ following two systems of equations are obtained:

Equations of the order $\left(\varepsilon^{0}\right)$

$$
\begin{aligned}
& \frac{\partial^{2}}{\partial T_{0}^{2}} u_{0}-\Omega \alpha 1 \frac{\partial}{\partial T_{0}} w_{0}+\alpha 2 u_{0}=0 \\
& \frac{\partial^{2}}{\partial T_{0}^{2}} w_{0}+\Omega \alpha 1 \frac{\partial}{\partial T_{0}} u_{0}+\alpha 2 w_{0}=0
\end{aligned}
$$

Equations of the order $\left(\varepsilon^{1}\right)$

$$
\begin{gathered}
\frac{\partial^{2}}{\partial T_{0}^{2}} u_{1}-\Omega \alpha 1 \frac{\partial}{\partial T_{0}} w_{1}+\alpha 2 u_{1}=\Omega \alpha 1 \frac{\partial}{\partial T_{0}} w_{0}-2 \frac{\partial^{2}}{\partial T_{0} \partial T_{1}} \\
\times u_{0}-\frac{\beta 1}{2}\left(u_{0}^{3}+u_{0} w_{0}^{2}\right)-\beta 2\left(u_{0}^{3}+u_{0} w_{0}^{2}\right) \\
-c 1 \frac{\partial}{\partial T_{0}} u_{0}+m 1 \Omega^{2} d_{1} f\left(l_{1}\right) \sin \Omega t \\
\frac{\partial^{2}}{\partial T_{0}^{2}} w_{1}+\Omega \alpha 1 \frac{\partial}{\partial T_{0}} u_{1}+\alpha 2 w_{1}=-\Omega \alpha 1 \frac{\partial}{\partial T_{0}} u_{0}-2 \frac{\partial^{2}}{\partial T_{0} \partial T_{1}} \\
\times w_{0}-\frac{\beta 1}{2}\left(w_{0}^{3}+w_{0} u_{0}^{2}\right)-\beta 2\left(w_{0}^{3}+w_{0} u_{0}^{2}\right)-c 1 \frac{\partial}{\partial T_{0}} w_{0} \\
+m 1 \Omega^{2} d_{1} f\left(l_{1}\right) \cos \Omega t
\end{gathered}
$$

The solution of the homogeneous system $\mathrm{O}\left(\varepsilon^{0}\right)$ is given as

$$
\begin{gathered}
u_{0}=A_{1}\left(T_{1}\right) e^{i \omega_{1} T_{0}}+A_{2}\left(T_{1}\right) e^{i \omega_{2} T_{0}}+[c c] \\
w_{0}=i A_{1}\left(T_{1}\right) e^{i \omega_{1} T_{0}}-i A_{2}\left(T_{1}\right) e^{i \omega_{2} T_{0}}+[c c]
\end{gathered}
$$

where $[c c]$ represents the complex conjugates of the previous terms in the same equation.

\subsection{Possible resonances and solvability conditions}

Substituting solution of $\mathrm{O}\left(\varepsilon^{0}\right)$ in $\mathrm{O}\left(\varepsilon^{1}\right)$ gives the following 2 equations

$$
\begin{gathered}
\frac{\partial^{2} u_{1}}{\partial T_{0}^{2}}-\Omega \alpha 1 \frac{\partial}{\partial T_{0}} w_{1}+\alpha 2 u_{1}=\left[-2(\beta 1+2 \beta 2)\left(A_{1}^{2} \bar{A}_{1}\right.\right. \\
\left.\left.+2 A_{1} A_{2} \bar{A}_{2}\right)+i \Omega \alpha 1 \frac{\partial A_{1}}{\partial T_{1}}-i c 1 \omega_{1} A_{1}-2 i \omega_{1} \frac{\partial A_{1}}{\partial T_{1}}\right] e^{\left(i \omega_{1} T_{0}\right)} \\
+\left[-2(\beta 1+2 \beta 2)\left(A_{2}^{2} \bar{A}_{2}+2 A_{1} \bar{A}_{1} A_{2}\right)\right. \\
\left.-i \Omega \alpha 1 \frac{\partial A_{2}}{\partial T_{1}}-i c 1 A_{2} \omega_{2}-2 i \omega_{2} \frac{\partial A_{2}}{\partial T_{1}}\right] e^{\left(i \omega_{2} T_{0}\right)} \\
-2(\beta 1+2 \beta 2) A_{1} A_{2}^{2} e^{i\left(\omega_{1}+2 \omega_{2}\right) T_{0}} \\
-2(\beta 1+2 \beta 2) A_{1}^{2} A_{2} e^{i\left(2 \omega_{1}+\omega_{2}\right) T_{0}} \\
-\frac{1}{2} i m 1 \Omega^{2} d_{1} f\left(l_{1}\right) e^{\left(i \Omega T_{0}\right)}+[c c]
\end{gathered}
$$

$$
\begin{gathered}
\frac{\partial^{2} w_{1}}{\partial T_{0}^{2}}+\Omega \alpha 1 \frac{\partial}{\partial T_{0}} u_{1}+\alpha 2 w_{1}=\left[-2 i(\beta 1+2 \beta 2)\left(A_{1}^{2} \bar{A}_{1}\right.\right. \\
\left.\left.+2 A_{1} A_{2} \bar{A}_{2}\right)-\Omega \alpha 1 \frac{\partial A_{1}}{\partial T_{1}}+c 1 \omega_{1} A_{1}-2 \omega_{1} \frac{\partial A_{1}}{\partial T_{1}}\right] e^{\left(i \omega_{1} T_{0}\right)} \\
+\left[-2 i(\beta 1+2 \beta 2)\left(A_{2}^{2} \bar{A}_{2}+2 A_{1} \bar{A}_{1} A_{2}\right)\right. \\
\left.-\Omega \alpha 1 \frac{\partial A_{2}}{\partial T_{1}}-c 1 A_{2} \omega_{2}-2 \omega_{2} \frac{\partial A_{2}}{\partial T_{1}}\right] e^{\left(i \omega_{2} T_{0}\right)} \\
+2 i(\beta 1+2 \beta 2) A_{1} A_{2}^{2} e^{i\left(\omega_{1}+2 \omega_{2}\right) T_{0}} \\
-2 i(\beta 1+2 \beta 2) A_{1}^{2} A_{2} e^{i\left(2 \omega_{1}+\omega_{2}\right) T_{0}} \\
+\frac{1}{2} m 1 \Omega^{2} d_{1} f\left(l_{1}\right) e^{\left(i \Omega T_{0}\right)}+[c c]
\end{gathered}
$$

A particular solution of (11) is assumed to be in the following form

$$
\begin{gathered}
u_{1}=P 1\left(T_{1}\right) e^{i \omega_{1} T_{0}}+Q 1\left(T_{1}\right) e^{i \omega_{2} T_{0}} \\
w_{1}=P 2\left(T_{1}\right) e^{i \omega_{1} T_{0}}+Q 2\left(T_{1}\right) e^{i \omega_{2} T_{0}}
\end{gathered}
$$

It can be observed from the particular solution and Equations (11) that there are two possible primary resonance conditions, $\Omega=\omega_{1}$ and $\Omega=\omega_{2}$. These 2 cases can be studied in a similar manner. The development for case $\Omega=\omega_{1}$ is presented in what follows, then results are briefly given for $\Omega=\omega_{2}$.

\subsection{Case of $\Omega=\omega_{1}$}

First the angular rotational speed of the rotor is expressed as $\Omega=\omega_{1}+\varepsilon \sigma_{1}$, where, $\sigma_{1}$ is a detuning parameter for controlling the nearness of $\Omega$ with $\omega_{1}$. There are some solvability conditions to be satisfied in order to obtain the solutions of Equations (11). The first step in determining these conditions is to substitute $\Omega=\omega_{1}+\varepsilon \sigma_{1}$ and the particular solution in these equations. Then the coefficients of $e^{i \omega_{1} T_{0}}$ and $e^{i \omega_{2} T_{0}}$ are equated on both sides of equations and the procedure given in [14] is followed to determine the solvability conditions.

The coefficients of $e^{i \omega_{1} T_{0}}$ for the Left Hand Side (L.H.S) of the resulting equations give,

$$
\begin{aligned}
& -P 1\left(\omega_{1}^{2}-\alpha 2\right)-i \omega_{1}^{2} \alpha 1 P 2 \\
& -P 2\left(\omega_{1}^{2}-\alpha 2\right)+i \omega_{1}^{2} \alpha 1 P 1
\end{aligned}
$$

The coefficients of $e^{i \omega_{2} T_{0}}$ for the L.H.S. of the resulting equations give,

$$
\begin{aligned}
& -Q 1\left(\omega_{2}^{2}-\alpha 2\right)-i \omega_{1} \omega_{2} \alpha 1 Q 2 \\
& -Q 2\left(\omega_{2}^{2}-\alpha 2\right)+i \omega_{2} \alpha 1 \alpha 1 Q 1
\end{aligned}
$$

The coefficients of $e^{i \omega_{1} T_{0}}$ for the Right Hand Side (R.H.S.) of the resulting equations give,

$$
\begin{aligned}
& -4 \beta 1 A_{1} A_{2} \bar{A}_{2}-4 \beta 2 A_{1}^{2} \bar{A}_{1}-2 \beta 1 A_{1}^{2} \bar{A}_{1}+i\left(-2 \omega_{1}+\alpha_{1} \omega_{1}\right) \\
& \times \frac{\partial A_{1}}{\partial T_{1}}-8 \beta 2 A_{1} A_{2} \bar{A}_{2}-i c 1 \omega_{1} A_{1}-\frac{1}{2} i m_{1} \omega_{1}^{2} d_{1} f\left(l_{1}\right) e^{i \sigma_{1} T_{1}}
\end{aligned}
$$


and

$$
\begin{aligned}
& -4 i \beta 1 A_{1} A_{2} \bar{A}_{2}-4 i \beta 2 A_{1}^{2} \bar{A}_{1}-2 i \beta 1 A_{1}^{2} \bar{A}_{1}+\left(2 \omega_{1}-\alpha_{1} \omega_{1}\right) \\
& \times \frac{\partial A_{1}}{\partial T_{1}}-8 i \beta 2 A_{1} A_{2} \bar{A}_{2}+c 1 \omega_{1} A_{1}+\frac{1}{2} m_{1} \omega_{1}^{2} d_{1} f\left(l_{1}\right) e^{i \sigma_{1} T_{1}}
\end{aligned}
$$

The coefficients of $e^{i \omega_{2} T_{0}}$ for the R.H.S. of the resulting equations give,

$$
\begin{aligned}
-4 \beta 1 A_{1} A_{2} \bar{A}_{1}-4 \beta 2 A_{2}^{2} \bar{A}_{2}-2 \beta 1 A_{2}^{2} \bar{A}_{2}-i\left(2 \omega_{2}+\alpha_{1} \omega_{1}\right) \\
\times \frac{\partial A_{2}}{\partial T_{1}}-8 \beta 2 A_{1} A_{2} \bar{A}_{1}-i c 1 \omega_{2} A_{2}
\end{aligned}
$$

and

$$
\begin{aligned}
4 i \beta 1 A_{1} A_{2} \bar{A}_{1}+4 i \beta 2 A_{2}^{2} \bar{A}_{2}+2 i \beta 1 A_{2}^{2} \bar{A}_{2}-\left(2 \omega_{2}+\alpha_{1} \omega_{1}\right) \\
\times \frac{\partial A_{2}}{\partial T_{1}}+8 i \beta 2 A_{1} A_{2} \bar{A}_{1}-c 1 \omega_{2} A_{2}
\end{aligned}
$$

The following two solvability conditions for this case are,

$$
\begin{aligned}
& \frac{\partial A_{1}}{\partial T_{1}}=-c 2 A_{1}^{2} \bar{A}_{1}-c 3 A_{1} A_{2} \bar{A}_{2}-c 5 A_{1}-c 4 e^{i \sigma_{1} T_{1}} \\
& \frac{\partial A_{2}}{\partial T_{1}}=-d 2 A_{2}^{2} \bar{A}_{2}-d 3 A_{1} \bar{A}_{1} A_{2}-d 5 A_{2}
\end{aligned}
$$

where $c 2, c 3, c 4, c 5, d 2, d 3, d 5$ are constants, given in Appendix B.

Substituting the solution of $A_{1}$ and $A_{2}$ in polar form $\left(A_{i}=\frac{1}{2} a_{i} e^{i \theta_{i}}, i=1 \ldots 2\right)$ in Equations (13) and separating the real and imaginary parts lead to the following system of equations:

$$
\begin{aligned}
& \frac{1}{2} \frac{\partial a_{1}}{\partial T_{1}}+\frac{1}{8} c 2 a_{1}^{3}+\frac{1}{8} c 3 a_{1} a_{2}^{2}+\frac{1}{2} c 5 a_{1}+c 4 \cos \left(-\theta_{1}+\sigma_{1} T_{1}\right)=0 \\
& \frac{1}{2} a_{1} \frac{\partial \theta_{1}}{\partial T_{1}}+c 4 \sin \left(-\theta_{1}+\sigma_{1} T_{1}\right)=0 \\
& \frac{1}{2} \frac{\partial a_{2}}{\partial T_{1}}+\frac{1}{8} d 2 a_{2}^{3}+\frac{1}{8} d 3 a_{1}^{2} a_{2}+\frac{1}{2} d 5 a_{2}=0 \\
& \frac{1}{2} a_{2} \frac{\partial \theta_{2}}{\partial T_{1}}=0
\end{aligned}
$$

The above system of four equations can be transformed to an autonomous system of equations using $\Gamma=-\theta_{1}+\sigma_{1} T_{1}$. Then the autonomous system is given as below,

$$
\begin{aligned}
& \frac{1}{2} \frac{\partial a_{1}}{\partial T_{1}}+\frac{1}{8} c 2 a_{1}^{3}+\frac{1}{8} c 3 a_{1} a_{2}^{2}+\frac{1}{2} c 5 a_{1}+c 4 \cos \Gamma=0 \\
& -\frac{1}{2} a_{1} \frac{\partial \Gamma}{\partial T_{1}}+\frac{1}{2} a_{1} \sigma_{1}+c 4 \sin \Gamma=0 \\
& \frac{1}{2} \frac{\partial a_{2}}{\partial T_{1}}+\frac{1}{8} d 2 a_{2}^{3}+\frac{1}{8} d 3 a_{1}^{2} a_{2}+\frac{1}{2} d 5 a_{2}=0 \\
& \frac{1}{2} a_{2} \frac{\partial \theta_{2}}{\partial T_{1}}=0 \\
& \frac{\partial \Gamma}{\partial T_{1}}=-\frac{\partial \theta_{1}}{\partial T_{1}}+\sigma_{1}
\end{aligned}
$$

Equations (15) show that $a_{2}=0$ is a solution. Equilibrium is also achieved in $\partial a_{2} / \partial T_{1}=0, \partial \Gamma / \partial T_{1}=0$. Eliminating the transformed phase shift $\Gamma$ from the modulation equations, the autonomous system above now reduces to two equations that can be resolved to give the following 6 th degree polynomial equation for plotting the resonant curves.

$$
c 4^{2}-\frac{1}{4} a_{\mathrm{E}}^{2}\left(c 5^{2}+\sigma_{1}^{2}\right) a_{\mathrm{E}}^{2}-\frac{1}{8} c 2 c 5 a_{\mathrm{E}}^{4}-\frac{1}{8} c 2^{2} a_{\mathrm{E}}^{6}=0
$$

The above polynomial is function of amplitude at equilibrium $a_{\mathrm{E}}$ and detuning parameter $\sigma_{1}$. Solution of these polynomials gives six solutions that are symbolically complicated expressions and are not reproduced here. Therefore these polynomials are treated numerically in the next section. Hence, the numerical solution will be used.

\subsection{Case of $\Omega=\omega_{2}$}

The angular rotational speed of the rotor is expressed as $\Omega=\omega_{2}+\varepsilon \sigma_{1}$. The procedure is quite similar to the previous one, then the solvability conditions lead to the two equations:

$$
\begin{aligned}
& \frac{\partial A_{1}}{\partial T_{1}}=-c 2 A_{1}^{2} \bar{A}_{1}-c 3 A_{1} A_{2} \bar{A}_{2}-c 5 A_{1} \\
& \frac{\partial A_{2}}{\partial T_{1}}=-d 2 A_{2}^{2} \bar{A}_{2}-d 3 A_{1} \bar{A}_{1} A_{2}-d 5 A_{2}-d 4 e^{i \sigma_{1} T_{1}}
\end{aligned}
$$

where $c 2, c 3, c 5, d 2, d 3, d 5$ are defined in Appendix B. By separating the real and imaginary parts of Equations (17), a system of four first order partial differential equations is obtained. Then the system of four equations can be transformed to a system of equations autonomous using $\Gamma=-\theta_{2}+\sigma_{1} T_{1}$.

The 6 th degree polynomial equation for plotting the resonant curves is

$$
d 4^{2}-\frac{1}{4} a_{\mathrm{E}}^{2}\left(d 5^{2}+\sigma_{1}^{2}\right)-\frac{1}{8} d 2 d 5 a_{\mathrm{E}}^{4}-\frac{1}{64} d 2^{2} a_{\mathrm{E}}^{6}=0
$$

The symbolic solutions are still complicated mathematical expressions. Hence, the numerical solution will be used again.

\section{Results and discussions}

\subsection{Resonant curves}

In the following section, the solutions of polynomial given by Equation (16), using numerical data given in Appendix B, over a range of $\sigma_{1}$ values are presented. The resonant curves are plotted which are of spring hardening type (Figs. 2 and 3). The linear analysis showed a resonance only at second critical speed but in the nonlinear analysis another resonance has appeared at first critical speed. The effect of nonlinearities has caused these curves 


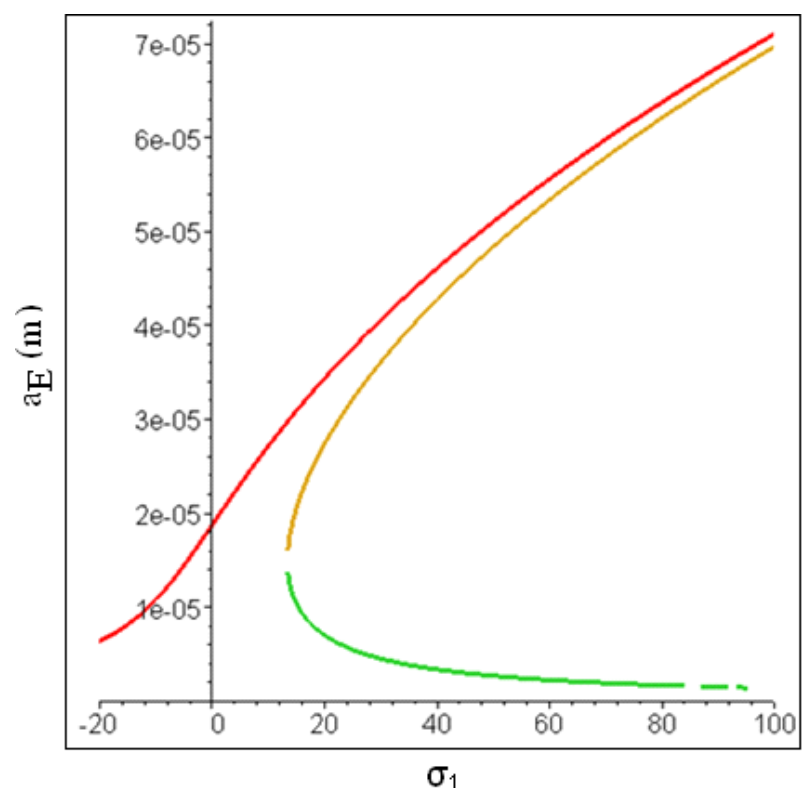

Fig. 2. Resonance curve at $\Omega=\omega_{1}$.

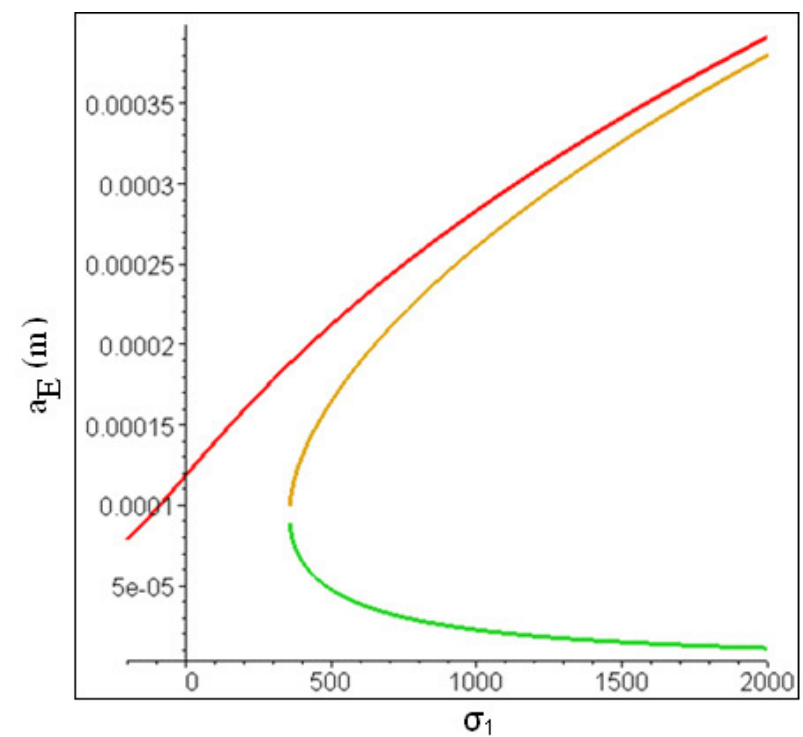

Fig. 3. Resonance curve at $\Omega=\omega_{2}$.

to bend towards right from the position of the linear response given in Appendix B Figure B.1b. It is interesting to note the horizontal plotting ranges of these curves. For the case $\Omega=\omega_{2}$ these curves are much more expanded as compared to those of $\Omega=\omega_{1}$. Also in this case the response amplitude is higher.

\subsection{Effect of various parameters}

In Equation (16) and (18) $c 2, c 4, c 5, d 2, d 4$ and $d 5$ are functions of $\alpha 1, \beta 1, \alpha 2, \beta 2$ and $\mathrm{m} 1$ (Appendix B) which further depend on parameters of geometry, material and mass unbalance. Therefore by changing these different parameters the behaviour of the rotor can be significantly

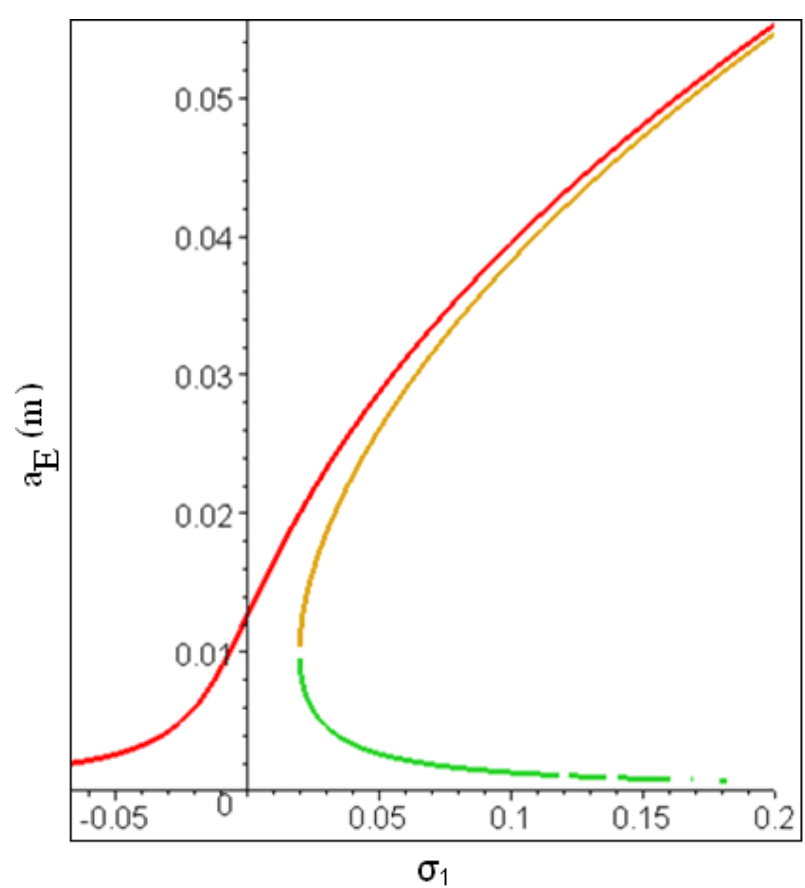

Fig. 4. Effect of $\beta 2=0$ at $\Omega=\omega_{1}$.

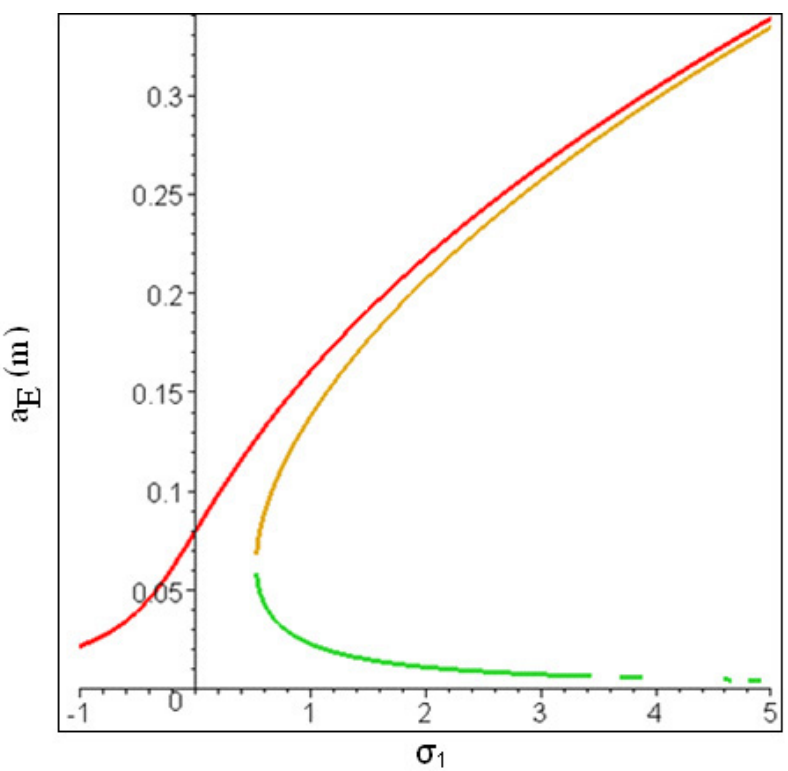

Fig. 5. Effect of $\beta 2=0$ at $\Omega=\omega_{2}$.

varied. Also $\beta 1$ and $\beta 2$ are due to higher order large deformations and effect of an axial dynamic force respectively. In Figures 4 to 9, the effect of change in the value of these parameters on the dynamic behavior of the rotor is graphically presented.

If the dynamic axial force is not taken into account that is by using $\beta 2=0$ in Appendix $\mathrm{B}$, the response will be as shown in Figures 4 and 5 . By comparing these figures with Figures 2 and 3, it is observed that in the absence of a dynamic axial force the response amplitude has increased. 


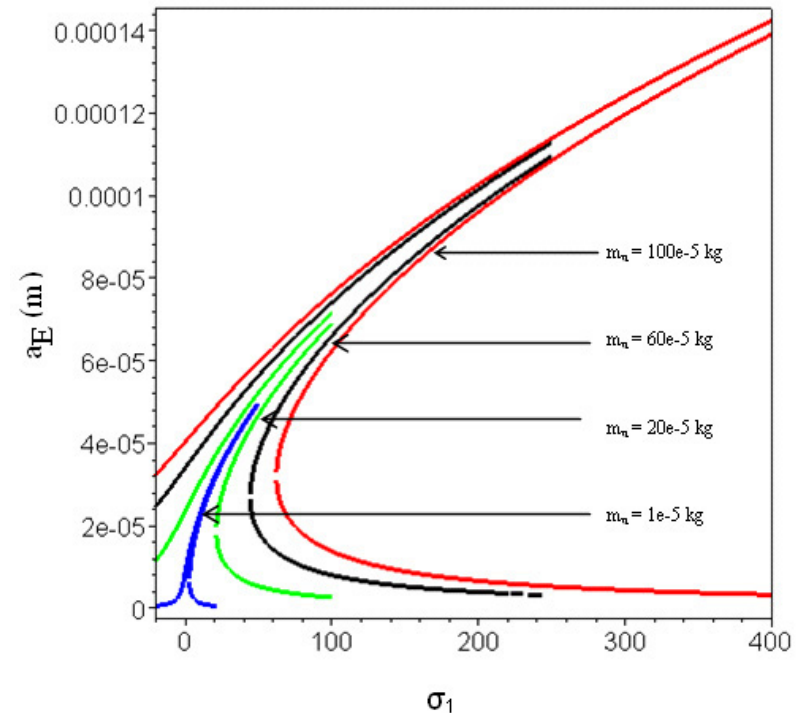

Fig. 6. Effect of variation in mass unbalance $m_{\mathrm{u}}$ at $\Omega=\omega_{1}$.

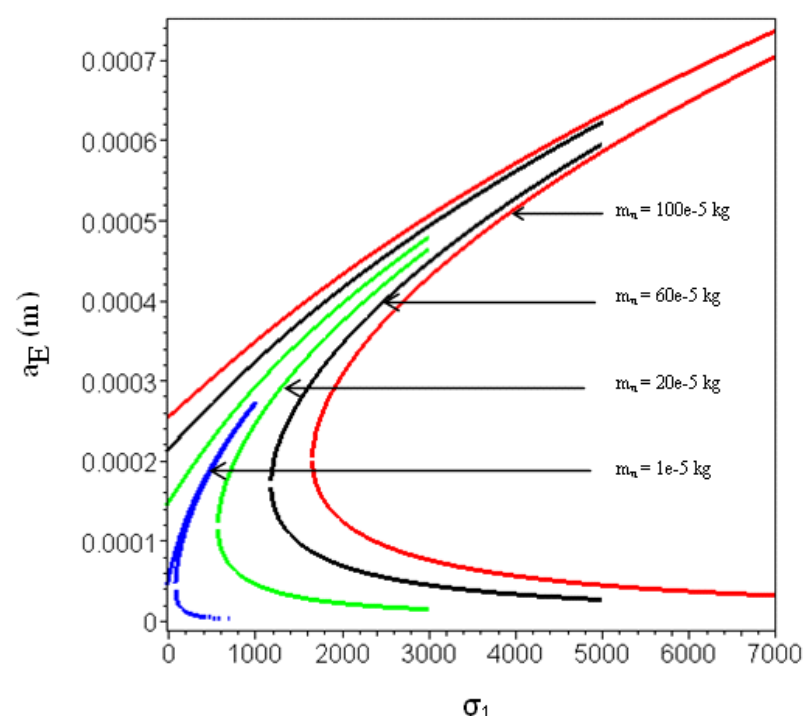

Fig. 7. Effect of variation in mass unbalance $m_{\mathrm{u}}$ at $\Omega=\omega_{2}$.

The effect of varying the magnitude of the unbalance mass is presented in Figures 6 and 7 corresponding to resonance cases $\Omega=\omega_{1}$ and $\Omega=\omega_{2}$ respectively. As the value of the unbalance mass is increased the resonant curves expand over a larger range of $\sigma_{1}$ values.

When the shaft of the rotor becomes slender i.e. its diameter is decreased, the response curves bend more strongly towards right. This indicates that the geometric nonlinearity becomes more effective. This can be observed in Figures 8 and 9.

\section{Conclusions}

Nonlinear dynamic behavior of the rotors due to geometric nonlinearity is analysed for the first mode. A mathematical model was developed using Hamilton's principle.

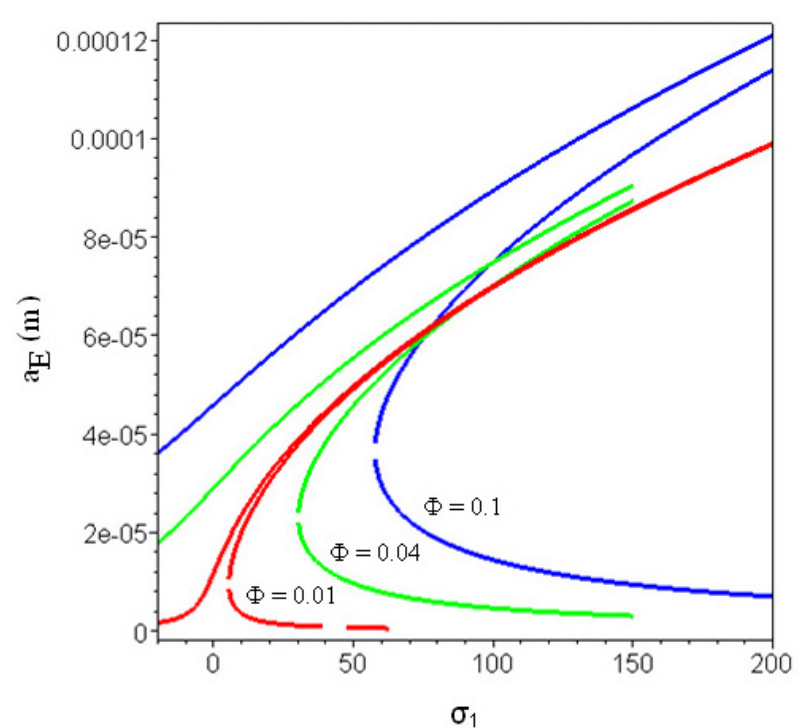

Fig. 8. Effect of variation in shaft diameter $\Phi$ at $\Omega=\omega_{1}$.

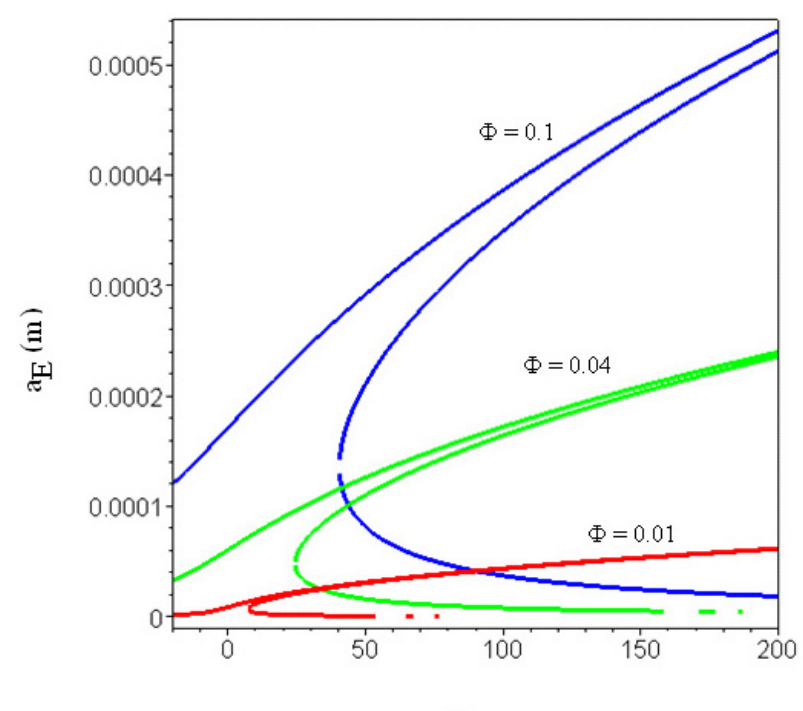

$\sigma_{1}$

Fig. 9. Effect of variation in shaft diameter $\Phi$ at $\Omega=\omega_{2}$.

The solution of the nonlinear equations of motion was obtained using the method of multiple scales. The results have shown that nonlinearities along with other effects like gyroscopic, rotary inertia and mass unbalance significantly influence the dynamics of the rotor system. Also nonlinearities have caused the resonance curves to be of a hard spring type. The variation in different parameters significantly affects the behavior of the rotor system. For example, the geometric nonlinearity becomes more apparent causing the resonant curves to bend strongly towards right, when the shaft diameter is decreased, i.e. when the shaft of the rotor becomes more slender.

The future perspectives of this work include the experimental validation of the results and the consideration of the effect of shear deformations (Timoshenko beam). 


\section{Appendix A}

\section{Strain energy of the shaft}

The displacements in the $x, y$ and $z$ directions of the beam are given below.

$$
u_{x}=u, u_{y}=-z \theta_{x}+x \theta_{z}, u_{z}=w
$$

The deformation in the $y$ direction can be shown to be

$$
\varepsilon_{y y}=\frac{-z \frac{\partial \theta_{x}}{\partial y}+x \frac{\partial \theta_{z}}{\partial y}}{\downarrow}+\underset{\substack{\downarrow \\ \varepsilon_{l}}}{\varepsilon_{n l} \text { (higher order deformations) }}
$$

The deformation energy can be given as:

$$
U_{\mathrm{s} 1}=\frac{1}{2} \int_{0}^{L} \int_{A}\left(\sigma_{y y} \varepsilon_{y y}\right) \mathrm{d} s \mathrm{~d} y
$$

Using the relation $\sigma_{y y}=E \varepsilon_{y y}$, the deformation energy can be written as:

$$
U_{\mathrm{s} 1}=\frac{1}{2} \int_{0}^{L} \int_{A} E \varepsilon_{y y} \varepsilon_{y y} \mathrm{~d} s \mathrm{~d} y=\frac{E}{2} \int_{0}^{L} \int_{A} \varepsilon_{y y}^{2} \mathrm{~d} s \mathrm{~d} y
$$

Using the deformation relations

$$
\begin{aligned}
U_{s 1}= & \frac{E}{2} \int_{0}^{L} \int_{A}\left(-z \frac{\partial \theta_{x}}{\partial y}+x \frac{\partial \theta_{z}}{\partial y}+\frac{1}{2} \theta_{x}^{2}+\frac{1}{2} \theta_{z}^{2}\right)^{2} \mathrm{~d} s \mathrm{~d} y \\
= & \frac{E}{2} \int_{0}^{L} \int_{A} \\
& \times\left\{\begin{array}{c}
z^{2}\left(\frac{\partial \theta_{x}}{\partial y}\right)^{2}+x^{2}\left(\frac{\partial \theta_{z}}{\partial y}\right)^{2}-2 x z\left(\frac{\partial \theta_{x}}{\partial y}\right) \\
\times\left(\frac{\partial \theta_{z}}{\partial y}\right)+\frac{1}{4} \theta_{x}^{4}+\frac{1}{2} \theta_{x}^{2} \theta_{z}^{2}+\frac{1}{4} \theta_{z}^{4} \\
-2\left(z \frac{\partial \theta_{x}}{\partial y}+x \frac{\partial \theta_{z}}{\partial y}\right)\left(\frac{1}{2} \theta_{x}^{2}+\frac{1}{2} \theta_{z}^{2}\right)
\end{array}\right\} \mathrm{d} s \mathrm{~d} y
\end{aligned}
$$

Several terms in the above equation vanish due to symmetry of the cross-section i.e.: $0=\int z \mathrm{~d} s, 0=\int x \mathrm{~d} s$.

Also, $I_{x}=\int_{A} z^{2} \mathrm{~d} s, I_{z}=\int_{A} x^{2} \mathrm{~d} A, I=I_{x} \stackrel{A}{=} I_{z} ; A=$ $\int_{S} \mathrm{~d} s=$ area of the cross section.

Thus the above equation becomes,

$$
\begin{aligned}
U_{\mathrm{s} 1}=\frac{E I}{2} \int_{0}^{L}\left\{\left(\frac{\partial \theta_{x}}{\partial y}\right)^{2}\right. & \left.+\left(\frac{\partial \theta_{z}}{\partial y}\right)^{2}\right\} \mathrm{d} y \\
& +\frac{E A}{2} \int_{0}^{L}\left(\frac{1}{4} \theta_{x}^{4}+\frac{1}{2} \theta_{x}^{2} \theta_{z}^{2}+\frac{1}{4} \theta_{z}^{4}\right) \mathrm{d} y
\end{aligned}
$$

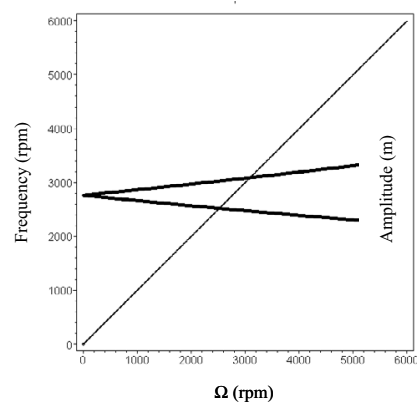

(a)

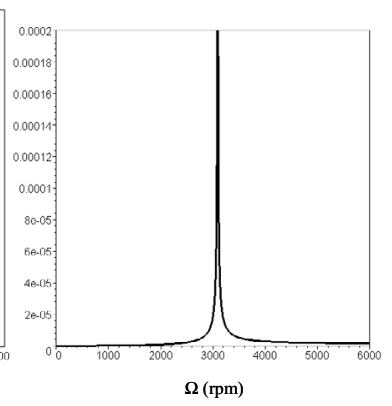

(b)
Fig. B.1. (a) Campbell diagram; (b) mass unbalance response.

\section{Appendix B}

\section{Linear behavior}

First the rotor is studied as a free undamped system to determine the natural frequencies of vibration and Campbell diagram is plotted to determine the critical speeds [2]. The 2 critical speeds $\left(\omega_{1}\right.$ and $\left.\omega_{2}\right)$ are found to be $2520 \mathrm{rpm}$ $(263 \mathrm{~Hz})$ and $3089 \mathrm{rpm}(323 \mathrm{~Hz})$.

\section{Constants}

Various constants incorporated in Equation (11) are given as

$$
c 2=\frac{C 2}{C 1}, c 3=\frac{C 3}{C 1}, c 5=\frac{C 5}{C 1}
$$

where $C 1, C 2, C 3, C 4$ and $C 5$ are given as

$$
\begin{aligned}
C 1 & =-2 \omega_{1}^{3}-\omega_{1}^{2} \omega_{2} \alpha 1+\alpha 1^{2} \omega_{1} \omega_{2}^{2}+2 \alpha 2 \omega_{1}-\alpha 1 \omega_{2} \alpha 2 \\
C 2 & =2 i(\beta 1+2 \beta 2)\left(\omega_{1}^{2}+\alpha 1 \omega_{1} \omega_{2}-\alpha 2\right) \\
C 3 & =4 i(\beta 1+2 \beta 2)\left(\omega_{1}^{2}+\alpha 2 \omega_{1} \omega_{2}-\alpha 2\right) \\
C 4 & =\frac{1}{2} m 1 d_{1} f\left(l_{1}\right)\left(\alpha 2 \omega_{1}^{2}-\omega_{1}^{4}+\omega_{1}^{4} \alpha 1\right)\left(\text { only for } \omega_{1}\right) \\
C 5 & =-c 1\left(\omega_{1}^{3}+\alpha 1 \omega_{1}^{2} \omega_{2}-\alpha 2 \omega_{1}\right)
\end{aligned}
$$

Also,

$$
d 2=\frac{D 2}{D 1}, \quad d 3=\frac{D 3}{D 1}, \quad d 4=\frac{D 4}{D 1}, \quad d 5=\frac{D 5}{D 1}
$$

where,

$$
\begin{aligned}
D 1 & =-\alpha 2 \omega_{2}(2+\alpha 2)-\alpha 1\left(\omega_{2}^{3}+\omega_{2}^{3} \alpha 1\right)+2 \omega_{2}^{3} \\
D 2 & =2 i(\beta 1+2 \beta 1)\left(-\omega_{2}^{2}+\alpha 1 \omega_{2}^{2}+\alpha 2\right) \\
D 3 & =4 i(\beta 1+2 \beta 2)\left(-\omega_{2}^{2}+\alpha 1 \omega_{2}^{2}+\alpha 2\right) \\
D 4 & =\frac{1}{2} m 1 d_{1} f\left(l_{1}\right)\left(\alpha 2 \omega_{2}^{2}-\omega_{2}^{4}+\omega_{2}^{4} \alpha 1\right)\left(\text { only for } \omega_{2}\right) \\
D 5 & =c 1\left(\omega_{1}^{3}-\alpha 1 \omega_{2}^{3}-\alpha 2 \omega_{2}\right)
\end{aligned}
$$




\section{Numerical data}

Following numerical data are used [2],

$L=0.4 \mathrm{~m}, R_{1}=0.01 \mathrm{~m}, R_{2}=0.15 \mathrm{~m}, h=0.03 \mathrm{~m}$, $m_{\mathrm{u}}=1 \times 10^{-4} \mathrm{~kg}$.

$I_{\mathrm{d} x}=0.0943 \mathrm{~kg} \cdot \mathrm{m}^{2}, I_{\mathrm{d} y}=0.186 \mathrm{~kg} \cdot \mathrm{m}^{2}$.

$\rho=7800 \mathrm{~kg} \cdot \mathrm{m}^{-3}, E=2 \times 10^{11} \mathrm{~N} \cdot \mathrm{m}^{-2}, c=$ 0.001 N.s.m ${ }^{-1}$.

Various parameters in the above expressions can be numerically given as: $\omega 1=258 \mathrm{~Hz}, \omega 2=323 \mathrm{~Hz}, f(l 1)=$ $0.8660, d 1=0.15, c 1=0.001$.

\section{References}

[1] J.S. Rao, Rotordynamics, 3rd edition, New Age International, India, 1996, p. 420, ISBN 8122409776

[2] M. Lalanne, G. Ferraris, Rotordynamics prediction in engineering, 2nd edition, John Wiley \& sons, 1998, p. 266, ISBN 9780471972884

[3] L.M. Adams, Rotating machinery vibrations from analysis to troubleshooting, Marcel Dekker Inc, New York, 2001, p. 354, ISBN 9780824702588

[4] T. Yamamoto, Y. Ishida, Linear and nonlinear rotordynamics: a modern treatment with applications, Wiley Series in Nonlinear Science, 2001, p. 348, ISBN: 9780471181750

[5] A. Muszynska, Rotordynamics, Taylor \& Francis, 2005, p. 1075 , ISBN 9780824723996

[6] G. Genta, Dynamics of rotating systems, Springer, New York, 2005, p. 685, ISBN 9780387209364

[7] J. Kicinski, Rotor dynamics, 2nd ed., IFFM Publishers, Gdansk, 2006, p. 539, ISBN 8372045429

[8] W. Batko, Z. Dąbrowski, J. Kiciñski, Nonlinear effects in technical diagnostics, Polish Academy of Science, Warsaw, 2008, p. 303, ISBN 9788372047489
[9] F. Ehrich, Observations of nonlinear phenomena in rotordynamics, J. Syst. Design Dyn. 2 (2008) 641-651

[10] Y. Ishida, I. Nagasaka, T. Inoue, S. Lee, Forced oscillations of a vertical continuous rotor with geometric nonlinearity, Nonlinear Dynamics 11 (1996) 107-120

[11] M. Duchemin, A. Berlioz, G. Ferraris, Dynamic behavior and stability of a rotor under base excitations, ASME J. Vib. Acoustics 128 (2006) 576-585

[12] N. Driot, C.H. Lamarque, A. Berlioz, Theoretical and experimental analysis of a base excited rotor, ASME J. Comput. Nonlinear Dyn. 1 (2006) 257-263

[13] M. Géradin, D. Rixen, Mechanical vibrations - Theory and application to structural dynamics, John Wiley \& Sons, 2nd ed., 1997, p. 425, ISBN 9780471975243

[14] A.H. Nayfeh, D.T. Mook, Nonlinear oscillations, Wiley and Sons, New York, 2007, p. 704, ISBN 9780471121428

[15] Nayfeh, A.H., Pai, P., Linear and nonlinear structural mechanics, Wiley and Sons, New York, 2004, p. 746, ISBN 9780471593560

[16] G. Michon, L. Manin, R.G. Parker, R. Dufour, Duffing oscillator with parametric excitation: analytical and experimental investigation on a belt-pulley system, ASME J. Computational Nonlinear Dynamics 3 (2008) 031001-1

[17] H. Yabuno, Y. Kunitho, T. Inoue, Y. Ishida, Nonlinear analysis of rotor dynamics by using method of multiple scales, IUTAM Symposium on Dynamics and Control of Nonlinear Systems with Uncertainty 2 (2007) 167-176

[18] R. Shad, G. Michon, A. Berlioz, Modeling and analysis of nonlinear rotordynamics due to high order deformation in bending, J. Appl. Math. Modeling 35 (2011) 2145-2159

[19] S. Hosseini, S. Khadem, Free vibration analysis of a rotating shaft with nonlinearities in curvature and inertia, Mech. Mach. Theory 44 (2009) 272-288 